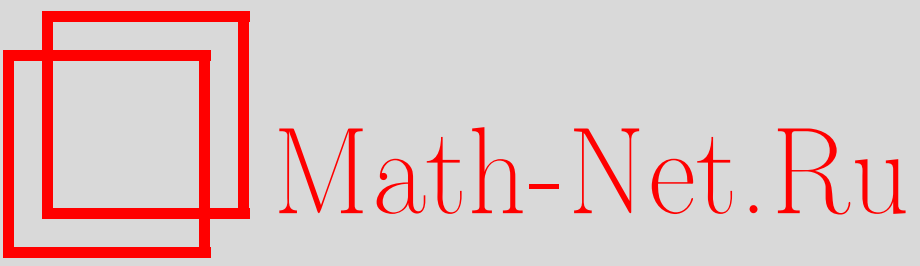

В. Н. Сачков, Случайные преобразования множеств с ограничениями на параметры. II, Матем. вопр. криптогр., 2012, том 3, выпуск 2, 97-116

DOI: https://doi.org/10.4213/mvk56

Использование Общероссийского математического портала Math-Net.Ru подразумевает, что вы прочитали и согласны с пользовательским соглашением

http://www.mathnet.ru/rus/agreement

Параметры загрузки:

IP : 54.197 .217 .227

26 апреля 2023 г., 13:37:04 
УДК 519.212.2

\title{
Случайные преобразования множеств с ограничениями на параметры. II
}

\author{
B. Н. Сачков \\ Академия криптографии Российской Федерации, Москва
}

Получено 20.V.2011

Рассматриваются графы преобразований регистров сдвига со случайной булевой функцией обратной связи с заданным весом. Получены выражения для условного распределения, среднего значения, дисперсии и биномиальных моментов числа начальных вершин таких графов. Указаны условия на цикловую структуру остовов случайных преобразований, при которых распределения числа циклических элементов при $n \rightarrow \infty$ сходятся к распределению Рэлея.

Для случайного преобразования с неподвижными точками найдено совместное предельное при $n \rightarrow \infty$ распределение числа образов фиксированной точки и длины контура графа преобразования.

Ключевые слова: графы преобразований регистров сдвига, случайные функции обратной связи, начальные вершины, циклические вершины, неподвижные точки, остовы графов случайных преобразований

\section{Random mappings of sets with restrictions on parameters. II}

\section{N. Sachkov}

Academy of Cryptography of Russian Federation, Moscow

\begin{abstract}
Graphs corresponding to the binary shift registers of length $l$ with a random Boolean feedback function of given weight are considered. Formulas for the conditional distribution, mean, variance and binomial moments of the number of initial vertices are obtained. Conditions ensuring the convergence of distributions of the number cycle vertices to the Rayleigh distribution are given. For random mapping with fixed points the limiting bivariate distribution of the number of successors and of the length of corresponding cycle for a given vertex is found.
\end{abstract}

Key words: shift register graphs, random feedback functions, source vertices, cyclic vertices, fixed points, cycles of random mapping graphs

Citation: Mathematical Aspects of Cryptography, 2012, vol. 3, no. 2, pp. 97-116 (Russian). 


\section{Введение}

В $§ 1$ работы рассматривается условное вероятностное распределение числа $\eta_{\ell}(\omega)$ начальных вершин графа преобразования регистра сдвига длины $l$ при заданном весе случайной булевой функции обратной связи. Найдены распределение, среднее значение, дисперсия и биномиальные моменты $\eta_{\ell}(\omega)$. Для биномиальных моментов получена асимптотическая формула и отмечена инвариантность ее вида с ростом порядка моментов. Найдено безусловное распределение числа $\eta_{\ell}$ начальных вершин преобразования регистра сдвига со случайной функцией обратной связи.

В $\S 2$ определяется совокупность $S_{k}(Q)$ подстановок степени $k$, удовлетворяющих некоторому комплексу $Q$ ограничений на цикловую структуру. В качестве примеров рассматриваются множества $S_{k}\left(Q^{(1)}\right)$ и $S_{k}\left(Q^{(2)}\right)$ подстановок, имеющих единичные циклы и не имеющих единичных циклов соответственно. Преобразование $n$-множества называется $Q$-преобразованием, если его остову соответствует $Q$-подстановка. Приводятся формулы для числа $Q$-подстановок $n$-множества с данными числами циклических элементов и компонент. Вводится понятие $\varepsilon$-инвариантности для совокупности $Q$-преобразований $n$-множества $W_{n}(Q)$. Показано, что при выполнении условия $\varepsilon$-инвариантности число $\xi_{n}(Q)$ циклических элементов при $n \rightarrow \infty$, как и при отсутствии ограничений, имеет распределение Рэлея.

В 3 рассматривается совокупность $F_{n}$ преобразований $n$-множества, содержащих неподвижные точки.

Орграф $\Gamma(\sigma)$ преобразования $\sigma \in F_{n}$ состоит из двух подграфов: корневого леса $L(\sigma)$ и орграфа $G(\sigma)$ без неподвижных точек. Для случайного равновероятного преобразования $\sigma \in F_{n}$ найдена асимптотика при $n \rightarrow \infty$ вероятности того, что фиксированная вершина $\Gamma(\sigma)$ принадлежит $G(\sigma)$. Пусть $\kappa_{n}$ - число образов фиксированной точки, принадлежащей $G(\sigma)$, и $\ell_{n}$ - длина соответствующего контура в $\Gamma(\sigma)$; для двумерной случайной величины $\left(\kappa_{n}, \ell_{n}\right)$ найдено условное распределение при условии, что точка принадлежит $G(\sigma)$. При $n \rightarrow \infty$ найдены предельные распределения для $\left(\frac{\kappa_{n}}{\sqrt{n}}, \frac{\ell_{n}}{\sqrt{n}}\right), \frac{\kappa_{n}}{\sqrt{n}}, \frac{\ell_{n}}{\sqrt{n}}$, а также точные и асимптотические формулы для средних значений $\mathbf{M} \kappa_{n}$ и $\mathbf{M} \ell_{n}$. 


\section{§ 1. Распределение числа начальных вершин орграфа регистрового преобразования для случайной функции обратной связи}

Регистровое преобразование $R$ регистра сдвига длины $l$ определяется отображением

$$
R:[G F(2)]^{\ell} \rightarrow[G F(2)]^{\ell}
$$

где

$$
R\left(\left(x_{0}, x_{1}, \ldots, x_{\ell-1}\right)\right)=\left(\left(x_{1}, x_{2}, \ldots, x_{\ell-1}, f\left(x_{0}, x_{1}, \ldots, x_{\ell-1}\right)\right),\right.
$$

$\left(x_{0}, x_{1}, \ldots, x_{\ell-1}\right)$ - двоичный вектор и $f\left(x_{0}, x_{1}, \ldots, x_{\ell-1}\right)$ - булева функции обратной связи регистра сдвига. В [2] показано, что если $\Gamma(R)$ - орграф преобразования $R$ и $G_{\ell}(r)$ - число регистров сдвига, для которых $\Gamma(R)$ имеет $r$ начальных вершин, то

$$
G_{\ell}(r)=\left|\left\{f:\left\|f\left(0, x_{1}, \ldots, x_{\ell-1}\right) \oplus f\left(1, x_{1}, \ldots, x_{\ell-1}\right) \oplus 1\right\|=r\right\}\right|,
$$

где $\|\varphi\|$ - вес булевой функции $\varphi$ и $\oplus$ - сложение в поле Галуа $G F(2)$.

Обозначим через $F_{\ell}(\omega)$ множество всех булевых функций от $\ell$ переменных веса $\omega$, а через $F_{\ell}-$ множество всех булевых функций от $\ell$ переменных. Зададим равномерные распределения на множествах $F_{\ell}(\omega)$ и $F_{\ell}$ и рассмотрим случайные величины

$$
\begin{aligned}
\eta_{\ell}(\omega) & =\left\|f\left(0, x_{1}, \ldots, x_{\ell-1}\right) \oplus f\left(1, x_{1}, \ldots, x_{\ell-1}\right) \oplus 1\right\|, f \in F_{\ell}(\omega), \\
\eta_{\ell} & =\left\|f\left(0, x_{1}, \ldots, x_{\ell-1}\right) \oplus f\left(1, x_{1}, \ldots, x_{\ell-1}\right) \oplus 1\right\|, f \in F_{\ell} .
\end{aligned}
$$

В соответствии с равенством (1.3) $\eta_{\ell}(\omega)$ - случайная величина, равная количеству начальных вершин орграфа $\Gamma(R)$ при случайном равновероятном выборе функции обратной связи $f$ из множества $F_{\ell}(\omega)$. Аналогично, $\eta_{\ell}$ определяет число начальных вершин $\Gamma(R)$ при случайном выборе $f$ из множества $F_{\ell}$. Ниже будут рассмотрены свойства распределений случайных величин $\eta_{\ell}(\omega)$ и $\eta_{\ell}$.

\section{1. Условное распределение}

Положим

$$
\begin{gathered}
\varphi=\varphi\left(x_{1}, \ldots, x_{\ell-1}\right)=f\left(0, x_{1}, \ldots, x_{\ell-1}\right), \Psi=\Psi\left(x_{1}, \ldots, x_{\ell-1}\right)=f\left(1, x_{1}, \ldots, x_{\ell-1}\right) . \text { Тогда } \\
\mathbf{P}\left(\eta_{\ell}(\omega)=r\right)=\mathbf{P}\left(\eta_{\ell}=r \mid\|f\|=\omega\right)= \\
=\sum_{\mu=0}^{\omega} \mathbf{P}(\|\varphi\|=\mu,\|\Psi\|=\omega-\mu) \mathbf{P}\left(\eta_{\ell}=r \mid\|\varphi\|=\mu,\|\Psi\|=\omega-\mu\right) .
\end{gathered}
$$


Очевидно, что

$$
\mathbf{P}(\|\varphi\|=\mu,\|\Psi\|=\omega-\mu)=\frac{\left(\begin{array}{c}
2^{\ell} \\
\mu
\end{array}\right)\left(\begin{array}{c}
2^{\ell-1} \\
\omega-\mu
\end{array}\right)}{\left(\begin{array}{l}
2^{\ell} \\
\omega
\end{array}\right)} .
$$

Для вычисления условной вероятности в сумме (1.5) применим метод включения-исключения:

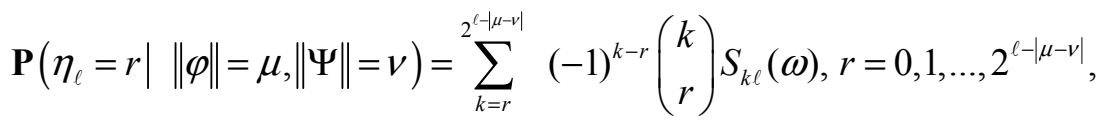

где

$$
S_{k \ell}=\frac{\left(\begin{array}{c}
2^{\ell-1} \\
k
\end{array}\right)}{\left(\begin{array}{c}
2^{\ell-1} \\
\mu
\end{array}\right)\left(\begin{array}{c}
2^{\ell-1} \\
\omega-\mu
\end{array}\right)} \sum_{t=0}^{k}\left(\begin{array}{l}
k \\
t
\end{array}\right)\left(\begin{array}{c}
2^{\ell-1}-k \\
\mu-t
\end{array}\right)\left(\begin{array}{c}
2^{\ell-1}-k \\
\omega-\mu-t
\end{array}\right) .
$$

Из формул (1.5)-(1.8) находим, что

$$
\begin{aligned}
& \mathbf{P}\left(\eta_{\ell}(\omega)=r\right)=\mathbf{P}\left(\eta_{\ell}=r \mid\|f\|=\omega\right)=
\end{aligned}
$$

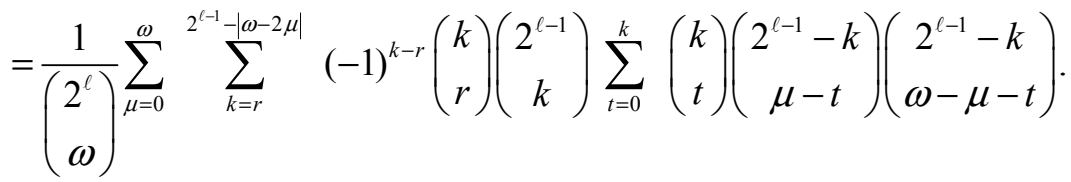

Изменим порядок первых двух суммирований в формуле (1.9):

$$
\begin{gathered}
\mathbf{P}\left(\eta_{\ell}(\omega)=r\right)=\mathbf{P}\left(\eta_{\ell}=r \mid\|f\|=\omega\right)= \\
=\frac{1}{\left(\begin{array}{c}
2^{\ell} \\
\omega
\end{array}\right)} \sum_{k=r}^{2^{\ell-1}-|\omega-2 \mu|}(-1)^{k-r}\left(\begin{array}{l}
k \\
r
\end{array}\right)\left(\begin{array}{c}
2^{\ell-1} \\
k
\end{array}\right) \sum_{\mu=0}^{\omega} \sum_{t=0}^{k}\left(\begin{array}{l}
k \\
t
\end{array}\right)\left(\begin{array}{c}
2^{\ell-1}-k \\
\mu-t
\end{array}\right)\left(\begin{array}{c}
2^{\ell-1}-k \\
\omega-\mu-t
\end{array}\right) .
\end{gathered}
$$

В формуле (1.10) рассмотрим отдельно второе и третье суммирования и поменяем их порядок:

$$
\sum_{\mu=0}^{\omega} \sum_{t=0}^{k}\left(\begin{array}{l}
k \\
t
\end{array}\right)\left(\begin{array}{c}
2^{\ell-1}-k \\
\mu-t
\end{array}\right)\left(\begin{array}{c}
2^{\ell-1}-k \\
\omega-\mu-t
\end{array}\right)=\sum_{t=0}^{k}\left(\begin{array}{l}
k \\
t
\end{array}\right) \sum_{\mu=t}^{\omega-t}\left(\begin{array}{c}
2^{\ell-1}-k \\
\mu-t
\end{array}\right)\left(\begin{array}{c}
2^{\ell-1}-k \\
\omega-\mu-t
\end{array}\right) .
$$


Для второй суммы в правой части равенства (1.11), используя известное соотношение для биномиальных коэффициентов, получаем

$$
\sum_{\mu=t}^{\omega-t}\left(\begin{array}{c}
2^{\ell-1}-k \\
\mu-t
\end{array}\right)\left(\begin{array}{c}
2^{\ell-1}-k \\
\omega-\mu-t
\end{array}\right)=\sum_{\mu=0}^{\omega-2 t} \sum_{t=0}^{k}\left(\begin{array}{c}
2^{\ell-1}-k \\
\mu
\end{array}\right)\left(\begin{array}{c}
2^{\ell-1}-k \\
\omega-2 t-\mu
\end{array}\right)=\left(\begin{array}{c}
2^{\ell}-2 k \\
\omega-2 t
\end{array}\right) .
$$

Из равенств (1.10) - (1.12) следует, что

$$
\begin{gathered}
\mathbf{P}\left(\eta_{\ell}(\omega)=r\right)=\mathbf{P}\left(\eta_{\ell}=r \mid\|f\|=\omega\right)= \\
=\frac{1}{\left(\begin{array}{c}
2^{\ell} \\
\omega
\end{array}\right)} \sum_{k=r}^{2^{\ell-1}}(-1)^{k-r}\left(\begin{array}{l}
k \\
r
\end{array}\right)\left(\begin{array}{c}
2^{\ell-1} \\
k
\end{array}\right) \sum_{t=0}^{k}\left(\begin{array}{l}
k \\
t
\end{array}\right)\left(\begin{array}{c}
2^{\ell}-2 k \\
\omega-2 t
\end{array}\right) .
\end{gathered}
$$

Условное распределение (1.13) можно представить в виде формулы включения-исключения:

$$
\mathbf{P}\left(\eta_{\ell}(\omega)=r\right)=\mathbf{P}\left(\eta_{\ell}=r \mid\|f\|=\omega\right)=\sum_{k=r}^{2^{\ell-1}}(-1)^{k-r}\left(\begin{array}{l}
k \\
r
\end{array}\right) \tilde{S}_{k \ell}(\omega),
$$

где

$$
\tilde{S}_{k \ell}(\omega)=\frac{\left(\begin{array}{c}
2^{\ell-1} \\
k
\end{array}\right)}{\left(\begin{array}{c}
2^{\ell} \\
\omega
\end{array}\right)} \sum_{t=0}^{k}\left(\begin{array}{l}
k \\
t
\end{array}\right)\left(\begin{array}{c}
2^{\ell}-2 k \\
\omega-2 t
\end{array}\right), k=0,1, \ldots,[\omega / 2] .
$$

Из формул (1.14), (1.15) и известного равенства $B_{k \ell}(\omega)=S_{k \ell}(\omega)$, $k=0,1 \ldots$, следует выражение для $k$-го биномиального момента случайной величины $\eta_{\ell}(\omega)$ :

$$
B_{k \ell}(\omega)=\frac{\left(\begin{array}{c}
2^{\ell-1} \\
k
\end{array}\right)}{\left(\begin{array}{c}
2^{\ell} \\
\omega
\end{array}\right)} \sum_{t=0}^{k}\left(\begin{array}{l}
k \\
t
\end{array}\right)\left(\begin{array}{c}
2^{\ell}-2 k \\
\omega-2 t
\end{array}\right) .
$$

Преобразуя правую часть равенства (1.16), получим другую формулу для $k$-го биномиального момента $\eta_{\ell}(\omega)$ :

$$
B_{k \ell}(\omega)=\left(\begin{array}{c}
2^{\ell-1} \\
k
\end{array}\right) \sum_{t=0}^{k}\left(\begin{array}{l}
k \\
t
\end{array}\right) \frac{(\omega)_{2 t}\left(2^{\ell}-\omega\right)_{2(k-t)}}{\left(2^{\ell}\right)_{2 k}} .
$$


Введем величину $\gamma=\frac{\omega}{2^{\ell}}$ и, исключая тривиальные случаи, будем предполагать, что $0<\gamma<1$. Из формулы (1.17) при выполнении неравенств $0 \leq t \leq \gamma 2^{\ell-1}, k-t \leq(1-\gamma) 2^{\ell-1}$ имеем оценку для порядков положительных биномиальных моментов $\eta_{\ell}(\omega)$

$$
0 \leq k \leq \min (\gamma, 1-\gamma) 2^{\ell-1} .
$$

Следующая лемма устанавливает ограничения на порядки таких биномиальных моментов $\eta_{\ell}(\omega)$, которые при $\ell \rightarrow \infty$ имеют предельные выражения, совпадающие с соответствующими биномиальными моментами биномиального распределения с параметрами $\left(2^{\ell-1}, \gamma^{2}+(1-\gamma)^{2}\right)$.

Лемма 1. При $\ell \rightarrow \infty$ и выполнении условия

$$
\frac{k}{2^{\ell / 2}} \rightarrow 0
$$

имеет место асимптотика

$$
B_{k \ell}(\omega)=\left(\begin{array}{c}
2^{\ell-1} \\
k
\end{array}\right)\left(\gamma^{2}+(1-\gamma)^{2}\right)^{k}(1+o(1))
$$

Асимптотика (1.20) следует из формулы (1.17) и неравенств

$$
1-\sum_{i=1}^{v} \delta_{i} \leq \prod_{i=1}^{v}\left(1-\delta_{i}\right) \leq e^{-\sum_{i=1}^{v} \delta_{i}}, \quad \text { где } 0<\delta_{i}<1, \quad i=1,2, \ldots, v .
$$

Для среднего $\mathbf{M} \eta_{\ell}(\omega)$ и дисперсии $\mathbf{D} \eta_{\ell}(\omega)$ случайной величины $\eta_{\ell}(\omega)$ имеем формулы

$$
\begin{gathered}
\mathbf{M} \eta_{\ell}(\omega)=\frac{2^{\ell-1}}{\left(2^{\ell}\right)_{2}}\left[\left(2^{\ell}-\omega\right)_{2}+(\omega)_{2}\right], \\
\mathbf{D} \eta_{\ell}(\omega)=\frac{\left(2^{\ell-1}\right)_{2}}{\left(2^{\ell}\right)_{4}}\left[\left(2^{\ell}-\omega\right)_{4}+2(\omega)_{2}\left(2^{\ell}-\omega\right)_{2}+(\omega)_{4}\right]+ \\
+\frac{2^{\ell-1}}{\left(2^{\ell}\right)_{2}}\left[\left(2^{\ell}-\omega\right)_{2}+(\omega)_{2}\right]-\left\{\frac{2^{\ell-1}}{\left(2^{\ell}\right)_{2}}\left[\left(2^{\ell}-\omega\right)_{2}+(\omega)_{2}\right]\right\}^{2} .
\end{gathered}
$$


Используя лемму 1 и формулы (1.21), для среднего и дисперсии $\eta_{\ell}(\omega)$ получаем асимптотики при $\ell \rightarrow \infty$

$$
\begin{gathered}
\mathbf{M} \eta_{\ell}(\omega)=2^{\ell-1}\left(\gamma^{2}+(1-\gamma)^{2}\right)\left(1+O\left(\frac{1}{2^{\ell}}\right)\right) \\
\mathbf{D} \eta_{\ell}(\omega)=2^{\ell-1}\left(\gamma^{2}+(1-\gamma)^{2}\right)\left(1-\gamma^{2}-(1-\gamma)^{2}\right)\left(1+O\left(\frac{1}{2^{\ell}}\right)\right) .
\end{gathered}
$$

Отсюда при $\gamma=\frac{1}{2}$ имеем

$$
\mathbf{M} \eta_{\ell}(\omega)=2^{\ell-2}\left(1+O\left(\frac{1}{2^{\ell}}\right)\right), \quad \mathbf{D} \eta_{\ell}(\omega)=2^{\ell-3}\left(1+O\left(\frac{1}{2^{\ell}}\right)\right) .
$$

Изучим теперь условия, при которых биномиальные моменты $B_{k \ell}(\omega)$ при $k \rightarrow \infty$ имеют асимптотики вида (1.20), когда $k$ растет с ростом $l$ не медленнее, чем $2^{\ell / 2}$. Это свойство биномиальных моментов $\eta_{\ell}(\omega)$ назовем acuмnтотической инвариантностью. Положим

$$
D(k, \ell, \gamma ; t)=\frac{(\omega)_{2 t}+\left(2^{\ell}-\omega\right)_{2(k-t)}}{\left(2^{\ell}\right)_{2 k}}, t=0,1, \ldots, k .
$$

Из формул (1.17) и (1.20) следует, что асимптотическая инвариантность биномиальных моментов имеет место, если условие (1.19) не выполнено и

$$
D(k, \ell, \gamma)=\sum_{t=0}^{k}\left(\begin{array}{l}
k \\
t
\end{array}\right) D(k, \ell, \gamma ; t)=\left(\gamma^{2}+(1-\gamma)^{2}\right)^{k}(1+o(1)) \quad \text { при } k \rightarrow \infty .
$$

Для определения условий существования асимптотики (1.25) при $k \geq 2^{\ell / 2}$ получим асимптотическое представление для чисел $D(k, \ell, \gamma ; t)$, заданных формулой (1.24).

Лемма 2. При $\ell \rightarrow \infty u \frac{k}{2^{\ell}} \rightarrow 0$ для любого натурального значения $m>1$ имеет место асимптотическое представление

$$
\begin{aligned}
D(k, \ell, \gamma ; t) & =\gamma^{2 t}(1-\gamma)^{2(k-t)} e^{-\frac{(t-\gamma k)^{2}}{\gamma(1-\gamma) 2^{\ell-1}}} \times \\
& \times \exp \left\{-\sum_{v=2}^{m-1} \frac{2^{v+1}}{v(v+1)\left(2^{\ell}\right)^{v}}\left[\frac{t^{v+1}}{\gamma^{v}}+\frac{(k-t)^{v+1}}{(1-\gamma)^{v}}-k^{\nu+1}\right]+O\left(\frac{k^{m+1}}{\left(2^{\ell}\right)^{m}}\right)\right\}
\end{aligned}
$$

с равномерной оценкой остаточного члена для всех $t, 0 \leq t \leq k$. 
Лемма 2 доказывается с использованием формулы Стирлинга и известной асимптотической формулы при $k \rightarrow \infty$

$$
\sum_{j=1}^{k-1} j^{v}=\frac{k^{v+1}}{v+1}+O\left(k^{v}\right)
$$

Рассмотрим сначала частный случай леммы 2 при $m=2$. Тогда

$$
D(k, \ell, \gamma ; t)=\gamma^{2 t}(1-\gamma)^{2(k-t)} e^{-\frac{(t-\gamma k)^{2}}{\gamma(1-\gamma) 2^{-1-1}}}\left(1+O\left(\frac{k}{\left(2^{\ell}\right)^{2 / 3}}\right)\right) .
$$

Отсюда следует, что для выполнения условия (1.25) достаточна при $\ell \rightarrow \infty$ равномерная для всех $0 \leq t \leq k$ сходимость

$$
\frac{(t-\gamma k)^{2}}{2^{\ell}} \rightarrow 0
$$

Ясно, что этот предел существует при выполнении условия (1.19) и не существует при $k \sim 2^{\ell / 2}$, например при $t=0$. Таким образом, $2^{\ell / 2}$ является граничным значением, для которого условие (1.28) не выполняется, равномерно для $0 \leq t \leq k$. Рассмотрим условие (1.28) для меньшего отрезка значений $t$ :

$$
\frac{(t-\gamma k)^{2}}{2^{\ell}} \rightarrow 0, t \in\left[\gamma k-k^{\varepsilon}, \gamma k+k^{\varepsilon}\right]
$$

где $t$ - любое натуральное число из отрезка $\left[\gamma k-k^{\varepsilon}, \gamma k+k^{\varepsilon}\right]$, представляющего собой окрестность точки $\gamma k$. Если существует такое $\varepsilon>0$, что выполнено условие (1.29), то соответствующая окрестность называется в-окрестностью инвариантности.

Если $\varepsilon$-окрестность существует, то сумму $D(k, \ell, \gamma)$ можно представить в виде двух сумм

$$
D(k, \ell, \gamma)=D^{(1)}(k, \ell, \gamma)+D^{(2)}(k, \ell, \gamma)
$$

где

$$
\begin{gathered}
D^{(1)}(k, \ell, \gamma)=\sum_{t \in\left[\gamma k \pm k^{\varepsilon}\right]}\left(\begin{array}{l}
k \\
t
\end{array}\right) \gamma^{2 t}(1-\gamma)^{2(k-t)}(1+o(1)), \\
D^{(2)}(k, \ell, \gamma)=\sum_{t \notin\left[\gamma k \pm k^{\varepsilon}\right]}\left(\begin{array}{l}
k \\
t
\end{array}\right) \gamma^{2 t}(1-\gamma)^{2(k-t)} e^{-\frac{(t-\gamma k)^{2}}{\gamma(1-\gamma) 2^{(-1}}}(1+o(1)) .
\end{gathered}
$$


Для оценки сумм (1.31) используем понятие ассоциированного распределения. В качестве такого распределения возьмем биномиальное распределение с параметрами $\left(k, \frac{\gamma^{2}}{\gamma^{2}+(1-\gamma)^{2}}\right)$, имеющее, как известно, среднее и дисперсию вида

$$
m=\frac{\gamma^{2} k}{\gamma^{2}+(1-\gamma)^{2}}, \sigma^{2}=\left[\frac{\gamma(1-\gamma) \sqrt{k}}{\gamma^{2}+(1-\gamma)^{2}}\right]^{2}
$$

С использованием ассоциированного распределения оценим вероятности

$$
\begin{gathered}
P^{(1)}(k, \ell, \gamma)=\left[\gamma^{2}+(1-\gamma)^{2}\right]^{-k} D^{(1)}(k, \ell, \gamma)= \\
=\sum_{t: t-\gamma k \mid \leq k^{\varepsilon}}\left(\begin{array}{l}
k \\
t
\end{array}\right)\left(\frac{\gamma^{2}}{\gamma^{2}+(1-\gamma)^{2}}\right)^{t}\left(\frac{(1-\gamma)^{2}}{\gamma^{2}+(1-\gamma)^{2}}\right)^{k-t}(1+o(1)), \\
P^{(2)}(k, \ell, \gamma)=\left[\gamma^{2}+(1-\gamma)^{2}\right]^{-k} D^{(2)}(k, \ell, \gamma) \leq \\
\leq \sum_{t: t-\gamma k \mid \leq k^{\varepsilon}}\left(\begin{array}{l}
k \\
t
\end{array}\right)\left(\frac{\gamma^{2}}{\gamma^{2}+(1-\gamma)^{2}}\right)^{t}\left(\frac{(1-\gamma)^{2}}{\gamma^{2}+(1-\gamma)^{2}}\right)^{k-t} .
\end{gathered}
$$

Из формулы (1.32) следует, что если при $k \rightarrow \infty$-окрестность инвариантности существует и выполнено условие

$$
P^{(1)}(k, \ell, \gamma) \rightarrow 1,
$$

то имеет место асимптотика (1.25), т. е. выполнено условие асимптотической инвариантности.

Следующая теорема указывает условие существования предела (1.34) и существования $\varepsilon$-окрестности инвариантности.

Теорема. Пусть $k, \ell \rightarrow \infty u \frac{k}{\left(2^{\ell}\right)^{2 / 3}} \rightarrow 0$. Тогда для соответствующей ع-окрестности инвариантности предел (1.34) существует тогда и только тогда, когда $\gamma=\frac{1}{2}$.

Из условия существования $\varepsilon$-окрестности инвариантности (1.29) следует выполнение соотношения $\frac{k^{2 \varepsilon}}{2^{\ell}} \rightarrow 0$. Поэтому для выполнения условия теоремы следует выбрать $\varepsilon=\frac{3}{4}$. 
Если $\gamma=\frac{1}{2}$, то ассоциированным является биномиальное распределение с параметрами $\left(k, \frac{1}{2}\right)$. Используя нормальное приближение для биномиального распределения при $k \rightarrow \infty$, из формулы (1.32) получаем, что

$$
P^{(1)}\left(k, \ell, \frac{1}{2}\right)=\sum_{t: t-k / 2 \mid \leq k^{3 / 4} / 2}\left(\begin{array}{l}
k \\
t
\end{array}\right) \frac{1}{2^{k}} \sim \frac{1}{\sqrt{2 \pi}} \int_{-k^{1 / 4}}^{k^{1 / 4}} e^{-\frac{u^{2}}{2}} d u .
$$

Следовательно, при $k \rightarrow \infty$ имеем

$$
P^{(1)}\left(k, \ell, \frac{1}{2}\right) \rightarrow 1, \quad P^{(2)}\left(k, \ell, \frac{1}{2}\right) \rightarrow 0,
$$

и, стало быть, выполнено условие асимптотической инвариантности (1.25) для биномиальных моментов $\eta_{\ell}(\omega)$.

Рассмотрим теперь случай, когда $\gamma \neq \frac{1}{2}, 0<\gamma<1$. Тогда

a) если $\gamma<\frac{1}{2}$, то $\gamma k>\frac{\gamma^{2} k}{\gamma^{2}+(1-\gamma)^{2}}$,

б) если $\gamma>\frac{1}{2}$, то $\gamma k<\frac{\gamma^{2} k}{\gamma^{2}+(1-\gamma)^{2}}$.

Поэтому при $\gamma<\frac{1}{2}$ и $\gamma>\frac{1}{2}$ для достаточно больших $k$ имеем, соответственно,

$$
\begin{aligned}
& \gamma k-k^{3 / 4}>\frac{\gamma^{2} k+\gamma(1-\gamma) k^{\frac{1}{2+\frac{1}{4}}}}{\gamma^{2}+(1-\gamma)^{2}}, \\
& \gamma k+k^{3 / 4}<\frac{\gamma^{2} k-\gamma(1-\gamma) k^{\frac{1}{2}+\frac{1}{4}}}{\gamma^{2}+(1-\gamma)^{2}} .
\end{aligned}
$$

Используя нормальное приближение при $k \rightarrow \infty$ для ассоциированного биномиального распределения, имеем

$$
\begin{gathered}
Q(k, \ell, \gamma)=\sum_{t:\left(\gamma^{2}+(1-\gamma)^{2}\right) t-\gamma^{2} k \mid \leq \gamma(1-\gamma) k^{3 / 4}}\left(\begin{array}{l}
k \\
t
\end{array}\right)\left(\frac{\gamma^{2}}{\gamma^{2}+(1-\gamma)^{2}}\right)^{t}\left(\frac{(1-\gamma)^{2}}{\gamma^{2}+(1-\gamma)^{2}}\right)^{k-t} \sim \\
\sim \frac{1}{\sqrt{2 \pi}} \int_{-k^{1 / 4}}^{k^{1 / 4}} e^{-\frac{u^{2}}{2}} d u .
\end{gathered}
$$


Отсюда следует предельное соотношение

$$
Q(k, \ell, \gamma) \rightarrow 1 \text { при } k \rightarrow \infty .
$$

Из формулы (1.32) имеем

$$
P^{(1)}(k, \ell, \gamma)=\sum_{t: t-\gamma k \mid \leq k^{3 / 4}}\left(\begin{array}{l}
k \\
t
\end{array}\right)\left(\frac{\gamma^{2}}{\gamma^{2}+(1-\gamma)^{2}}\right)^{t}\left(\frac{(1-\gamma)^{2}}{\gamma^{2}+(1-\gamma)^{2}}\right)^{k-t} .
$$

Ясно, что $P^{(1)}(k, \ell, \gamma) \leq Q(k, \ell, \gamma)$, и в силу неравенств $(1.37)$ и (1.38) интервалы $\left[\gamma k-k^{3 / 4}, \gamma k+k^{3 / 4}\right]$ значений $t$ как при $\gamma<\frac{1}{2}$, так и при $\gamma>\frac{1}{2}$ находятся в области больших уклонений нормального приближения ассоциированного биномиального распределения. Следовательно, в обоих случаях при $k \rightarrow \infty$ имеем

$$
P^{(1)}(k, \ell, \gamma) \rightarrow 0
$$

Стало быть, асимптотическая инвариантность для биномиальных моментов $\eta_{\ell}(\omega)$ указанных в условиях теоремы порядков не имеет места. Теорема доказана.

Рассмотрим теперь более общий случай, когда при $\ell \rightarrow \infty$ выполнено условие $\frac{k}{2^{\ell}} \rightarrow 0$. Тогда из условия существования $\varepsilon$-окрестности инвариантности (1.29) следует соотношение $\frac{k^{2 \varepsilon}}{2^{\ell}} \rightarrow 0$. Это соотношение означает, что $\varepsilon$-окрестность может существовать только при $\varepsilon \leq \frac{1}{2}$.

В асимптотическом представлении $D(k, \ell, \gamma, t)$ вида (1.26) подставим максимальные значения $t$ из отрезка $\left[\gamma k-k^{\varepsilon}, \gamma k+k^{\varepsilon}\right]$ в члены представления и определим допустимые значения $\varepsilon$. Для главного члена допустимым является $\varepsilon \leq \frac{1}{2}$. Для общего члена асимптотического разложения (1.26) в квадратных скобках при $t \in\left[\gamma k-k^{\varepsilon}, \gamma k+k^{\varepsilon}\right]$ при $k \rightarrow \infty$ имеем представление

$$
\frac{\left(\gamma k \pm k^{\varepsilon}\right)^{v+1}}{\gamma^{\nu}}+\frac{\left((1-\gamma) k \mp k^{\varepsilon}\right)^{v+1}}{(1-\gamma)^{\nu}}-k^{\nu+1}=\frac{\nu(\nu+1)}{2 \gamma(1-\gamma)} k^{\nu-1+2 \varepsilon}\left(1+O\left(\frac{1}{k^{1-\varepsilon}}\right)\right) .
$$


При $\varepsilon \leq \frac{1}{2}$ из соотношения (1.40) следует оценка для суммы в асимптотическом разложении при $t \in\left[\gamma k-k^{\varepsilon}, \gamma k+k \varepsilon\right]$ с остаточным членом

$$
-\frac{1}{\gamma(1-\gamma)} \sum_{v=2}^{m-1} \frac{k^{\nu-1+2 \varepsilon}}{\left(2^{\ell-1}\right)^{v}}\left(1+O\left(\frac{1}{k^{1-\varepsilon}}\right)\right) \text {. }
$$

Эта сумма при $k \rightarrow \infty$ и $\frac{k^{2 \varepsilon}}{2^{\ell}} \rightarrow 0$ при любом фиксированном $m$ стремится к нулю. Отметим, что остаточный член в формуле (1.26) в данном случае имеет порядок $O\left(\left(\frac{k}{2^{\ell}}\right)^{m}\right)$.

\section{2. Безусловное распределение числа начальных точек.}

Обозначим через $\eta_{\ell}$ число начальных точек $\Gamma(R)$ в предположении, что функция обратной связи $f$ случайно и равновероятно выбирается из множества булевых функций от $l$ переменных. Тогда из формулы

$$
\mathbf{P}\left(\eta_{\ell}=r\right)=\sum_{\omega=0}^{2^{\ell}} \mathbf{P}(\|\varphi\|=\omega) \mathbf{P}\left(\eta_{\ell}=r \mid\|f\|=\omega\right)
$$

и равенства (1.9) следует, что

$$
\begin{gathered}
\mathbf{P}\left(\eta_{\ell}=r\right)=\sum_{\omega=0}^{2^{\ell}} \frac{\left(\begin{array}{c}
2^{\ell} \\
\omega
\end{array}\right)}{2^{2^{\ell}}} \cdot \frac{1}{\left(\begin{array}{c}
2^{\ell} \\
\omega
\end{array}\right)} \sum_{k=r}^{2^{\ell-1}}(-1)^{k-r}\left(\begin{array}{l}
k \\
r
\end{array}\right)\left(\begin{array}{c}
2^{\ell-1} \\
k
\end{array}\right) \sum_{t=0}^{k}\left(\begin{array}{l}
k \\
t
\end{array}\right)\left(\begin{array}{c}
2^{\ell}-2 k \\
\omega-2 t
\end{array}\right), \\
r=0,1, \ldots, 2^{\ell-1} .
\end{gathered}
$$

Меняя порядок суммирования, получаем

$$
\mathbf{P}\left(\eta_{\ell}=r\right)=\frac{1}{2^{2^{\ell}}} \sum_{k=r}^{2^{\ell-1}}(-1)^{k-r}\left(\begin{array}{l}
k \\
r
\end{array}\right)\left(\begin{array}{c}
2^{\ell-1} \\
k
\end{array}\right) \sum_{t=0}^{k}\left(\begin{array}{l}
k \\
t
\end{array}\right) \sum_{\omega=2 t}^{2^{\ell}-2 k+2 t}\left(\begin{array}{c}
2^{\ell}-2 k \\
\omega-2 t
\end{array}\right) .
$$

После замены переменной в последней сумме, используя известные тождества для биномиальных коэффициентов, получаем окончательный вид распределения $\eta_{\ell}$ :

$$
\mathbf{P}\left(\eta_{\ell}=r\right)=\sum_{k=r}^{2^{\ell-1}}(-1)^{k-r}\left(\begin{array}{c}
k \\
r
\end{array}\right)\left(\begin{array}{c}
2^{\ell-1} \\
k
\end{array}\right) \frac{1}{2^{k}}, r=0,1, \ldots, 2^{\ell-1} .
$$


Из этой формулы получаем выражение для $k$-го биномиального момента $\eta_{\ell}$ :

$$
B_{k \ell}=\left(\begin{array}{c}
2^{\ell-1} \\
k
\end{array}\right) \frac{1}{2^{k}}, \quad k=0,1, \ldots, 2^{\ell-1} .
$$

Отсюда следуют формулы для среднего и дисперсии $\eta_{\ell}$ :

$$
\mathbf{M} \eta_{\ell}=2^{\ell-2}, \mathbf{D} \eta_{\ell}=2^{\ell-3} .
$$

Производящая функция биномиальных моментов имеет вид

$$
B_{\ell}(x)=\left(1+\frac{x}{2}\right)^{2^{\ell-1}} .
$$

Следовательно, для производящей функции $\eta_{\ell}$ имеем формулу

$$
P_{\ell}(x)=\left(\frac{1+x}{2}\right)^{2^{\ell-1}},
$$

из которой вытекает, что $\eta_{\ell}$ имеет биномиальное распределение с параметрами $\left(2^{\ell-1}, \frac{1}{2}\right)$ и что при $\ell \rightarrow \infty$ случайная величина

$$
\tilde{\eta}_{\ell}=\frac{\eta_{\ell}-2^{\ell-2}}{\sqrt{2^{\ell-3}}}
$$

асимптотически нормальна с параметрами $(0,1)$.

\section{§ 2. $Q$-преобразования конечных множеств и є-инвариантность}

Обозначим через $S_{k}(Q)$ множество подстановок степени $k$, удовлетворяющих некоторому комплексу ограничений $Q$ на цикловую структуру. Подстановку $s \in S_{k}(Q)$ будем называть $Q$-подстановкой. Частным случаем $Q$-подстановок являются известные $A$-подстановки, длины циклов которых являются элементами множества $A$. Другие примеры $Q$-подстановок представляют $S_{k}\left(Q^{(1)}\right)$ и $S_{k}\left(Q^{(2)}\right):$ множество подстановок, имеющих хотя бы один единичный цикл, и множество подстановок без единичных циклов соответственно. Известно, что

$$
\left|S_{k}\left(Q^{(1)}\right)\right|=k !-h_{k},\left|S_{k}\left(Q^{(2)}\right)\right|=h_{k}, \text { где } h_{k}=k ! \sum_{j=0}^{k} \frac{(-1)^{j}}{j !}
$$

причем $h_{k}$ есть целое число, ближайшее к величине $k ! e^{-1}$. 
Можно показать, что если $C_{k j}\left(Q^{(1)}\right)$ и $C_{k j}\left(Q^{(2)}\right)$ - числа подстановок степени $k$ с $j$ циклами с ограничениями $Q^{(1)}$ и $Q^{(2)}$ соответственно, то

$$
C_{k j}\left(Q^{(1)}\right)=\sum_{i=1}^{j}(-1)^{i-1}\left(\begin{array}{c}
k \\
i
\end{array}\right) C(k-i, j-i), \quad C_{k j}\left(Q^{(2)}\right)=\sum_{i=0}^{j}(-1)^{i}\left(\begin{array}{c}
k \\
i
\end{array}\right) C(k-i, j-i),
$$

где $C(k, j)=|S(k, j)|$ и $S(k, j)$ - числа Стирлинга первого рода.

Преобразование $n$-множества будем называть $Q$-преобразованием, если его остов является $Q$-подстановкой.

Обозначим через $U_{n}(k, j ; Q)$ число $Q$-отображений с $k$ циклическими элементами и $j$ компонентами. Тогда так же, как для $A$-отображений, доказывается, что

$$
U_{n}(k, j ; Q)=\left(\begin{array}{c}
n-1 \\
k-1
\end{array}\right) n^{n-k} C_{k j}(Q) .
$$

Если $U_{n}(k ; Q)$ и $U_{n j}(Q)$ - числа $Q$-преобразований с $k$ циклическими элементами и $j$ компонентами соответственно, то

$$
\begin{gathered}
U_{n}(k ; Q)=\left(\begin{array}{c}
n-1 \\
k-1
\end{array}\right) n^{n-k} C_{k}(Q), k=0,1, \ldots, \\
U_{n j}(Q)=\sum_{k=j}^{n}\left(\begin{array}{c}
n-1 \\
k-1
\end{array}\right) n^{n-k} C_{k j}(Q), j=0,1, \ldots,
\end{gathered}
$$

где $C_{k}(Q)$ и $C_{k j}(Q)$ - числа $Q$-подстановок степени $k$ и $Q$-подстановок с $j$ циклами соответственно.

Отсюда следует, что

$$
U_{n}\left(k ; Q^{(1)}\right)=\left(\begin{array}{c}
n-1 \\
k-1
\end{array}\right) n^{n-k}\left(k !-h_{k}\right), U_{n}\left(k ; Q^{(2)}\right)=\left(\begin{array}{c}
n-1 \\
k-1
\end{array}\right) n^{n-k} h_{k}
$$

и, соответственно,

$$
\begin{aligned}
& U_{n j}\left(Q^{(1)}\right)=\sum_{k=j}^{n}\left(\begin{array}{l}
n-1 \\
k-1
\end{array}\right) n^{n-k} \sum_{i=1}^{j}(-1)^{i-1}\left(\begin{array}{l}
k \\
i
\end{array}\right) C(k-i, j-i), \\
& U_{n j}\left(Q^{(2)}\right)=\sum_{k=j}^{n}\left(\begin{array}{c}
n-1 \\
k-1
\end{array}\right) n^{n-k} \sum_{i=0}^{j}(-1)^{i}\left(\begin{array}{c}
k \\
i
\end{array}\right) C(k-i, j-i) .
\end{aligned}
$$


Пусть $W_{n}(Q)$ - совокупность всех таких преобразований $n$-множества, что любой элемент $W_{n}(Q)$ является $Q$-преобразованием. Это означает, что остов любого преобразования является $Q$ - подстановкой некоторой степени $j, 1 \leq j \leq n$.

Обозначим через $C_{j}(Q)$ число $Q$-подстановок степени $j$, определяющих остовы $Q$-преобразований.

Будем говорить, что совокупность $W_{n}(Q)$ удовлетворяет условию $\varepsilon$-инвариантности, если для любого $0<\varepsilon<\frac{1}{6}$ равномерно для всех таких $j$, что $n^{\frac{1}{2}-\varepsilon} \leq j \leq n^{\frac{1}{2}+\varepsilon}$, при $n \rightarrow \infty$ выполнено равенство

$$
\frac{C_{j}(Q)}{j !}=d(1+o(1))
$$

где $0<d \leq 1$. Величина $d$ называется коэффициентом инвариантности.

Примерами совокупностей преобразований $n$-множества со свойством $\varepsilon$-инвариантности могут служить преобразования, остовы которых определяются подстановками, либо имеющими единичные циклы, либо не содержащими единичных циклов. В первом случае $d=1-e^{-1}$, во втором случае $d=e^{-1}$.

Известно, что для числа циклических элементов $\xi_{n}$ случайного преобразования $n$-множества распределение $\xi_{n} / \sqrt{n}$ при $n \rightarrow \infty$ сходится к распределению Рэлея. Докажем следующую теорему.

Теорема. Для числа циклических элементов $\xi_{n}(Q)$ случайного равновероятного преобразования $\sigma \in W_{n}(Q)$, где $W_{n}(Q)$ удовлетворяет условию $\varepsilon$-инвариантности, при $n \rightarrow \infty$ равномерно по всем таким $u=o\left(n^{1 / 6}\right)$, что u $\sqrt{n}$ - натуральное число, имеет место соотношение

$$
\mathbf{P}\left(\frac{\xi_{n}(Q)}{\sqrt{n}}=u\right)=u e^{-\frac{u^{2}}{2}} \frac{1}{\sqrt{n}}(1+o(1)) .
$$

Для $0<\varepsilon<\frac{1}{6}$ при $n \rightarrow \infty$ имеем оценки

$$
\begin{aligned}
& \frac{1}{n^{n}} \sum_{v=1}^{n^{\frac{1}{2} \varepsilon}} U_{n}(v: Q) \leq \sum_{v=1}^{n^{\frac{1}{2} \varepsilon}} \frac{v(n)_{v}}{n^{v+1}} \leq \frac{1}{n^{2 \varepsilon}}, \\
& \frac{1}{n} \sum_{\substack{\frac{1}{2^{+}+\varepsilon+1} \\
v=n^{2}}}^{n} U_{n}(v: Q) \leq \sum_{\substack{\frac{1}{2^{2}+\varepsilon+1} \\
v}}^{n} \frac{v}{n} e^{-\frac{v^{2}}{2 n}} \leq n^{\frac{1}{2}+\varepsilon} e^{-\frac{n^{2} \varepsilon}{2}} .
\end{aligned}
$$

2012, T. 3, № 1, С. 97-116 
В силу условия $\varepsilon$-инвариантности имеем

$$
\begin{gathered}
\frac{1}{n^{n}} U_{n}(k ; Q)=d \frac{k(n)_{k}}{n^{k+1}}(1+o(1)), \\
\frac{1}{n^{n}} \sum_{v=n^{\frac{1}{2}}}^{n^{\frac{1}{2}+\varepsilon}} \frac{v(n)_{V}}{n^{v+1}} U_{n}(k ; Q)=d \sum_{v=n^{\frac{1}{2} \varepsilon}}^{\frac{1}{n^{2}+\varepsilon}} \frac{v(n)_{v}}{n^{v+1}}(1+o(1)) .
\end{gathered}
$$

Следовательно,

$$
\mathbf{P}\left(\xi_{n}(Q)=k\right)=\frac{\frac{k(n)_{k}}{n^{k+1}}}{\sum_{\substack{\frac{1}{n^{2}}-\varepsilon \\ n^{\frac{1}{2}+\varepsilon}}} \frac{v(n)_{v}}{n^{\nu+1}}}(1+o(1)) .
$$

Так как $\sum_{v=1}^{n} \frac{v(n)_{v}}{n^{v+1}}=1 \quad$ и при $n \rightarrow \infty \quad$ имеем $\frac{k(n)_{k}}{n^{k+1}}=\frac{k}{n} e^{-\frac{k(k-1)}{2 n}} \times$ $\times\left(1+O\left(\frac{k^{3}}{n^{2}}\right)\right)$, то, полагая $u=\frac{k}{\sqrt{n}}$, при $u=o\left(n^{1 / 6}\right)$ получаем указанное в теореме предельное распределение Рэлея для случайной величины $\xi_{n}(Q)$.

Преобразования $n$-множества, остовы которых либо содержат единичные циклы, либо не имеют единичных циклов, обладают свойством $\varepsilon$-инвариантности. Поэтому из теоремы вытекает следствие.

Следствие. Распределения случайных величин $\frac{\xi_{n}\left(Q^{(1)}\right)}{\sqrt{n}} u \frac{\xi_{n}\left(Q^{(2)}\right)}{\sqrt{n}} n p u$ $n \rightarrow \infty$ сходятся к распределению Рэлея, указанному в теореме.

\section{§ 3. Характеризация преобразований с неподвижными элементами}

Пусть $X$ - конечное множество, $|X|=n, \sigma_{n}$ - множество всех преобразований $\sigma: X \rightarrow X, F_{n}-$ множество преобразований с неподвижными элементами и $G_{n}-$ совокупность преобразований без неподвижных элементов. Если $\Gamma(\sigma)$ - орграф преобразования $\sigma \in \sigma_{n}$, то компоненты $\Gamma(\sigma)$ представляют собой объединение орграфов $L(\sigma)$ и $G(\sigma)$, где $L(\sigma)$ - корневой лес и $G(\sigma)$ - орграф, не содержащий деревьев. Очевидно, что

$$
\left|F_{n}\right|=n^{n}-(n-1)^{n},\left|G_{n}\right|=(n-1)^{n} .
$$


Для $\sigma \in F_{n}$ орграф $\Gamma(\sigma)$ содержит в качестве компонент корневые деревья.

Для числа лесов $L_{n}$ с $n$ вершинами имеем формулу

$$
L_{n}=(n+1)^{n-1} .
$$

Исходя из структуры $\Gamma(\sigma)$ при $\sigma \in F_{n}$, имеем

$$
\left|F_{n}\right|=\sum_{k=1}^{n}\left(\begin{array}{c}
n \\
m
\end{array}\right) L_{m}\left|G_{n-m}\right| .
$$

Из этого равенства следует тождество

$$
n^{n}=\sum_{k=0}^{n}\left(\begin{array}{l}
n \\
m
\end{array}\right)(m+1)^{m-1}(n-m-1)^{n-m}
$$

справедливость которого вытекает также из тождества Абеля [2].

На множестве $F_{n}$ зададим равномерное вероятностное распределение. Для случайного отображения $\sigma \in F_{n}$ обозначим через $\tilde{P}_{n}$ вероятность того, что фиксированная вершина $x \in X$ орграфа $\Gamma(\sigma)$ принадлежит $G(\sigma)$. Так как $\tilde{P}_{n}=1-P_{n}$, где $P_{n}$ - вероятность того, что $x$ принадлежит лесу $L(\sigma)$, то, используя известную формулу [2]

$$
P_{n}=\frac{(n-1) !}{n^{n}-(n-1)^{n}} \sum_{m=0}^{n-1} \frac{n^{m}}{m !}
$$

и асимптотику при $n \rightarrow \infty$

находим, что

$$
P_{n}=\frac{1}{1-e^{-1}} \sqrt{\frac{\pi}{2 n}}(1+o(1))
$$

$$
\tilde{P}_{n}=1-\frac{1}{1-e^{-1}} \sqrt{\frac{\pi}{2 n}}(1+o(1)) .
$$

Рассмотрим двумерную случайную величину $\left(\kappa_{n}, \ell_{n}\right)$, где $\kappa_{n}-$ число образов фиксированной точки $x \in X$, принадлежащей $G(\sigma)$, для случайного преобразования $\sigma \in F_{n}$ и $\ell_{n}$ - длина соответствующего контура. Условное распределение $\left(\kappa_{n}, \ell_{n}\right)$ при условии, что точка $x \in X$ принадлежит $G(\sigma)$, имеет вид

$$
\begin{gathered}
\mathbf{P}\left(\kappa_{n}=k, \ell_{n}=j\right)=\frac{1}{\left(n^{n}-(n-1)^{n}\right)\left(1-P_{n}\right)} \times \\
\times \sum_{m=1}^{n-k}\left(\begin{array}{c}
n-1 \\
m
\end{array}\right)(m+1)^{m-1}(n-m-1)_{k-1}(n-m-1)^{n-m-k} .
\end{gathered}
$$


Отсюда следует, что

$$
\mathbf{P}\left(\kappa_{n}=k, \ell_{n}=j\right)=\frac{(n-1)_{k-1}}{\left(n^{n}-(n-1)^{n}\right)\left(1-P_{n}\right)} \sum_{m=1}^{n-k}\left(\begin{array}{c}
n-k \\
m
\end{array}\right)(m+1)^{m-1}(n-m-1)^{n-m-k} .
$$

Применяя к правой части этого равенства тождество Абеля, находим, что

$$
\mathbf{P}\left(\kappa_{n}=k, \ell_{n}=j\right)=\frac{(n)_{k}}{n^{k+1}\left(1-\left(1-\frac{1}{n}\right)^{n}\right)\left(1-P_{n}\right)}, 1 \leq j \leq k \leq n .
$$

Из этого равенства вытекает следующая теорема.

Теорема 1. При $n \rightarrow \infty$ условное предельное распределение $\left(\frac{\kappa_{n}}{\sqrt{n}}, \frac{\ell_{n}}{\sqrt{n}}\right)$, при условии, что фиксированная точка $x \in X$ принадлежст $G(\sigma)$, имеет вид

$$
\mathbf{P}\left(\frac{\kappa_{n}}{\sqrt{n}}=x, \frac{\ell_{n}}{\sqrt{n}}=y\right)=\frac{1}{1-e^{-1}} e^{-\frac{x^{2}}{2}} \frac{1}{n}(1+o(1))
$$

для всех таких $0<y \leq x, x=o\left(n^{1 / 6}\right)$, что $x \sqrt{n}, y \sqrt{n}-$ иелье числа. Соответствующие одномерные распределения имеют следующие выражения:

$$
\begin{gathered}
\mathbf{P}\left(\frac{\kappa_{n}}{\sqrt{n}}=x\right)=\frac{1}{1-e^{-1}} e^{-\frac{x^{2}}{2}} \frac{1}{\sqrt{n}}(1+o(1)), \\
\mathbf{P}\left(\frac{\ell_{n}}{\sqrt{n}}=y\right)=\frac{\sqrt{2 \pi}}{1-e^{-1}}(1-\Phi(y)) \frac{1}{\sqrt{n}}(1+o(1)), \quad \text { где } \Phi(y)=-\frac{1}{\sqrt{2 \pi}} \int_{-\infty}^{y} e^{-\frac{u^{2}}{2}} d u .
\end{gathered}
$$

Формула для двумерного предельного распределения следует из точного распределения $\left(\kappa_{n}, \ell_{n}\right)$, а одномерные распределения получаются интегрированием из двумерного распределения.

Из одномерного распределения интегрированием получаем выражение для функции распределения $\kappa_{n} / \sqrt{n}$. Для всех таких $x=o\left(n^{1 / 6}\right)$, что $x \sqrt{n} \geq 0$ - целое число, имеем

$$
\mathbf{P}\left(\frac{\kappa_{n}}{\sqrt{n}}=x\right)=\frac{1}{1-e^{-1}} e^{-\frac{x^{2}}{2}} \frac{1}{\sqrt{n}}(1+o(1)) .
$$

Производящую функцию распределения $\kappa_{n}$

$$
f_{n}(x)=\left(1-\left(\frac{1}{n}\right)^{n}\right)^{-1} \sum_{k=1}^{n} \frac{k(n)_{k}}{n^{k+1}} x^{k}
$$


можно привести к виду

$$
f_{n}(x)=\left(1-\left(\frac{1}{n}\right)^{n}\right)^{-1}\left[1+(x-1)(n-1) ! \sum_{k=0}^{n-1} \frac{1}{k !}\left(\frac{x}{n}\right)^{n-k-1}\right] \text {. }
$$

Вычисляя производные $f_{n}(x)$ при $x=1$, получаем формулы для среднего и дисперсии $\kappa_{n}$ :

$$
\begin{gathered}
\mathbf{M} \kappa_{n}=\left(1-\left(1-\frac{1}{n}\right)^{n}\right)^{-1} \frac{n !}{n^{n}} \sum_{k=0}^{n-1} \frac{n^{k}}{k !} \\
\mathbf{D} \kappa_{n}=\left(1-\left(1-\frac{1}{n}\right)^{n}\right)^{-1}\left[2 n-\frac{n !}{n^{n}} \sum_{k=0}^{n-1} \frac{n^{k}}{k !}\right]-\left(1-\left(1-\frac{1}{n}\right)^{n}\right)^{-2}\left(\frac{n !}{n^{n}} \sum_{k=0}^{n-1} \frac{n^{k}}{k !}\right)^{2} .
\end{gathered}
$$

Отсюда

$$
\begin{aligned}
& \mathbf{M} \kappa_{n}=\frac{1}{1-e^{-1}} \sqrt{\frac{\pi n}{2}}(1+o(1)), n \rightarrow \infty, \\
& \mathbf{D} \kappa_{n}=\frac{n}{1-e^{-1}}\left[2-\frac{\pi}{2\left(1-e^{-1}\right)}\right](1+o(1)) .
\end{aligned}
$$

Из точного распределения случайной величины $\ell_{n}$

$$
\mathbf{P}\left(\ell_{n}=j\right)=\frac{1}{1-\left(1-\frac{1}{n}\right)^{n}} \sum_{k=j}^{n} \frac{(n)_{k}}{n^{k+1}}, j=1, \ldots, n,
$$

следует выражение для среднего значения

$$
\mathbf{M} \ell_{n}=\frac{1}{1-\left(1-\frac{1}{n}\right)^{n}} \cdot \frac{1}{2} \sum_{k=1}^{n} k(k+1) \frac{(n)_{k}}{n^{k+1}} .
$$


Отсюда вытекает асимптотическая формула

$$
\mathbf{M} \ell_{n}=\frac{1}{1-e^{-1}} \cdot \frac{1}{2} \sqrt{\frac{\pi n}{2}}(1+o(1)), n \rightarrow \infty .
$$

Если $d_{n}-$ случайная величина, равная расстоянию от точки $x$ до цикла, то из равенства

$$
\mathbf{M} \kappa_{n}=\mathbf{M} \ell_{n}+\mathbf{M} d_{n}
$$

следует, что

$$
\mathbf{M} d_{n}=\frac{1}{1-e^{-1}} \cdot \frac{1}{2} \sqrt{\frac{\pi n}{2}}(1+o(1)), n \rightarrow \infty .
$$

Отметим, что безусловное распределение числа образов фиксированной точки для случайного преобразования без неподвижных точек рассматривалось в статье [3].

\section{Список литературы}

1. Феллер В. Введение в теорию вероятностей и ее приложения. - М.: Мир, 1967.

2. Сачков В. Н. Случайные преобразования множеств с ограничениями на параметры. I // Математические вопросы криптографии. - 2012. - Т. 3. Вып. 1. - С. 125-144.

3. Harris $B$. Probability distributions related to random mappings // Ann. Math. Stat. - 1960. - V. 31. No. 4. - P. 1045-1062. 